\title{
Day case versus inpatient tonsillectomy in southern Iran: a cost-effectiveness study
}

\author{
Mohammad Faramarzi ${ }^{1}$, Mozhgan Fardid ${ }^{2}$, Reza Kaboodkhani ${ }^{1}$, Mehrnoosh Emadi ${ }^{1}$, Javad \\ Shahmohamadi ${ }^{1}$, and Khosro Keshavarz ${ }^{1}$ \\ ${ }^{1}$ Shiraz University of Medical Sciences \\ ${ }^{2}$ Shahrood University of Medical Sciences
}

April 28, 2020

\begin{abstract}
Background: Day-case tonsillectomy (DCT) compared with inpatient tonsillectomy has increasingly become a norm for many patients undergoing elective surgeries. Unjustified stays of tonsillectomy can be avoided by day case surgery which consequently reduces treatment costs. The aim of this study was to determine the cost and effectiveness of DCT or outpatient operation in comparison with inpatient tonsillectomy. Methods: This study was a cost-effectiveness study which was performed on 300 patients. Patients were randomly divided into two groups: day case $(\mathrm{n}=150)$ and inpatient $(\mathrm{n}=150)$. Consequences used in model included incidence of bleeding, blood transfusion and re-operation frequency within two weeks after surgery and also the patients' pain during 24 hours after surgery. This study was conducted from the social perspective; therefore, direct and indirect costs are included in the study. One-way sensitivity analysis was conducted to measure the uncertainty effects of the parameters. The collected data was analyzed using software Tree-Age and Excel 2016. Results: The results showed that DCT was less costly and more cost effective than the inpatient one. Mean total costs in day case and inpatient were $\$ 915.1$ and $\$ 1227.9$, respectively. Besides, the mean effectiveness was 0.921 and 0.914 percent, respectively. Also, one-way sensitivity analysis proved the robustness of the results of the study. Conclusion: The results showed that DCT is a cost-effective strategy and can be suggested as a good alternative for a wide range of patients after tonsillectomy. According to the result of our study, supporting day case surgery for tonsillectomy cases can significantly reduce the financial burden. Keywords: Day Case Tonsillectomy, outpatient tonsillectomy, Inpatient tonsillectomy, Cost-effectiveness analysis
\end{abstract}

\section{Introduction}

Tonsillectomy is one of the most common surgeries in the field of otolaryngology. Annually more than 530,000 surgeries are performed on children younger than 15 years in the United States(1). Tonsillectomy is ranked second among pediatric outpatient surgeries and may have complications such as bleeding, pain and even death(2). Its mortality rate due to bleeding, airway obstruction or anesthesia complications is 1 in 15,000(3). Specifically, the rate mortality of post-tonsillectomy is about $2-4 \%$. Studies show that approximately $1.3 \%$ of children experience prolonged hospitalization and delayed discharge after tonsillectomy. Besides, more than $3.9 \%$ of them require readmission due to secondary complications. The main reasons for prolonged hospitalization or readmission are complications such as post-tonsillectomy bleeding, fever, moderate to severe pain, and nausea(4). Also, ENT specialists often prefer to hospitalize the patient after tonsillectomy, considering the risk of tonsillectomy complications $(5,6)$.

Long-term hospitalization in addition to exposing the patient to a variety of nosocomial infections and psychological stress such as anxiety imposes heavy costs and threatens the individual's mental and physical health $(7,8)$. Day case surgery has significantly grown within past two decades, following the development of short-term anesthesia and new methods(9). The term of day case surgery refers to surgery operation 
in hospital in day in which patients are prepared for scheduled, non-emergency surgical procedures and discharged within eight hours of surgery. Day case surgery was founded by Nickel (1864-1921)(10). In some countries such as Canada and the United States, day case surgery has increasingly become the norm for all patients undergoing elective surgery compared with inpatient surgery. It is performed for almost $90 \%$ of all surgery operations, but in many other countries it is less common (10). Day case surgery covers a wide range of surgical procedures from local anesthesia to general anesthesia(11). Applying day case surgeries can reduce treatment costs and saves the resources(7). However, it is important to recognize whether day case surgery can meet the patient needs or not due to the fact that not all patients can be treated by day case surgery $(11,12)$. Therefore, this study aimed to determine the most cost-effective method for patients by examining the cost and effectiveness aspects of day case surgery in comparison with inpatient surgery after tonsillectomy.

\section{Materials \& Methods:}

This was a cross-sectional cost-effectiveness study on a sample of 300 patients (children 3 and above) from May 2017 to April 2018 in DK [removed for blind peer review]*. Patients were randomly divided into two groups: day case $(\mathrm{n}=150)$ and hospitalization $(\mathrm{n}=150)$. Surgery was performed by an academic otolaryngologist and the operation, anesthesia and used drugs were similar in both groups. Incidence of bleeding, blood transfusion, and frequency of re-operation were recorded within two weeks after surgery. To compare the incidence of bleeding incidence after surgery, and the incidence of blood transfusion and the incidence of re-operation in two groups of DCT and inpatient, Chi-square test was used. Besides, Paired t-test was used to compare the pain in two groups of DCT and inpatient. Besides, patients' pain Level was measured by VAS (Visual Analysis Scale) within the first 24 hours after surgery was recorded. A data collection form was also used to collect cost and effectiveness data.

Inclusion criteria were children with the age of 3-15 years, referred to tonsillectomy because of recurrent tonsillectomy or hypertrophy of tonsils, having no other illnesses such as heart disease, lung disease, diabetes, anemia, metabolic disease, congenital syndrome; having a personal car at home, parents' presence at home, 30-minute distance by car from hospital, being able to use a mobile phone, have an educated family by least a high school education and patients' voluntary participation.

\section{Treatment costs}

Costs were collected from societal perspective, so the costs included direct medical (DMC) and non-medical costs (DNMC) as well as indirect costs (IC). DMC included patient's hoteling in hospital, medications used for hospital patients, laboratory and pathology tests, surgical costs, and other health services for each patient in the inpatient and day case tonsillectomy group. DNMC included the transportation costs for patients and their companions, the money paid for accommodation and meals by patients' companions for both hospitalized and day-to-day tonsillectomy in hospital. IC also included the patients' average of absence days from work, the companions' average of absence days from work to take care of their patients and the lost productivity.

Costs calculation was according to tariffs of 2018 in the international dollar (purchasing power parity (PPP)). Furthermore, the exchange rate of each PPP $\$$ was equal to 12800 rials (IRR)(13).

\section{Clinical inputs}

Blood transfusions and the incidence of bleeding within two weeks after surgery, re-operation and the severity of pain within 24 hours after surgery were considered as the outcomes of this study. To calculate pain score after surgery, the visual analogue scale (VAS) was used. It has a $10-\mathrm{cm}$ horizontal or vertical line, one side indicating the absence of pain (0) and the other side indicating the most severe pain (10), on which the patients put a mark based on their pain severity(14). Patients were contacted three times $(6,12$, and 24 hours) from the first day after surgery to respond to the questionnaire at VAS. During the first two weeks after surgery, if the bleeding occurs, the nurse recommends family to refer to the surgeon to check for the presence of the bleeding and its severity. In addition, the nurse should record the bleeding rate and pain 
score using a questionnaire.

\section{Cost-effectiveness analysis}

Incremental cost-effectiveness ratio (ICER) was used.

$$
I C E R=\frac{\text { cost Day Case Tonsillectomy }- \text { cost inpatient }}{\text { Effectiveness DayCase Tonsillectomy }- \text { Effectiveness inpatient }}
$$

Data analysis was performed using Tree Age and Excel 2016 software. Besides, chi-square and independent t-tests $(\mathrm{p}<0.05)$ were used for data analysis.

\section{Sensitivity analysis}

A one-way sensitivity analysis was performed to measure the uncertainty effects of parameters on model results. The threshold for developing countries, according to the World Health Organization (WHO), is one to three times the Gross Domestic Product (GDP) per capita that was about $\$ 21011$ PPP at 2018 for Iran, according to the World Bank report $(15,16)$.

\section{Results}

Totally 300 patients were included in this study. To compare the gender between two groups of DCT and inpatient, the chi-square test was used. There was no significant difference in the scores of two groups (p $=0.002$ ). Chi-square test was used to compare patients' education in two groups, however, there was no significant difference between two groups $(\mathrm{p}=0.362)$. Furthermore, 96 patients $(32 \%)$ were employed and had a significant role in the community but 81 patients $(27 \%)$ were not employed because most of them were children. There was no retired person in the study. The chi-square test was used to compare the employment of patients in two groups and it showed no significant difference between two groups $(\mathrm{p}=0.270)$.

\section{Costs}

According to Table 2, the mean of DMC in DCT group (\$ 874.6) was lower than inpatient group (\$1151.5). A significant difference was observed in DMC between two groups $(\mathrm{p}<0.01)$. Besides, the mean of DNMC in DCT group (\$18.7) was lower than inpatient group (\$27.0) and the difference between the mean total costs for patients and their companions food in two groups was $\$ 9$ and a significant difference was seen between two groups $(\mathrm{p}<0.01)$. The total mean of IC, including the days absent from work and lost productivity for patients and their companions in the DCT group (\$21.7) was lower than in the inpatient group (\$49.3). Thus, in general, the results showed that in DCT group, DMC, DNMC and IC were all lower than inpatient group. The total average cost in DCT group (\$915.1) was also significantly lower than inpatient group $(\$$ 1227.9).

\section{Effectiveness}

According to Table 3, about $2 \%$ of patients of DCT group and $2.6 \%$ of patients of inpatient group developed post-tonsillectomy bleeding. There was no significant difference in the incidence of bleeding after surgery between two groups $(\mathrm{P}=0.684)$. In DCT group, only $1.3 \%$ patients needed blood transfusion whereas in inpatient group only 1 patient $(0.6 \%)$ required blood transfusion after surgery. There was no significant difference in the incidence of blood transfusion between two groups of DCT and inpatient $(\mathrm{p}=0.624)$. In DCT group, 2 patients (1.3\%) and in inpatient group 3 patients $(2 \%)$ had re-operation due to bleeding. The results showed no significant difference in the incidence of re-operation among the patients of DCT and inpatient group $(\mathrm{p}=1.000)$. Pain score was recorded 3 times 24 hours after surgery. The results revealed no significant difference in the pain of two groups (DCT and inpatient) for 6 hours, 12 hours and 24 hours after surgery $(\mathrm{P}=0.240)$.

\section{Cost-effectiveness analysis}


The effectiveness of DCT group, which did not have bleeding, blood transfusion, re-operation, and pain, was greater than or equal to the inpatient group (Table 4). For patients with less bleeding and requiring re-operation, the effectiveness of DCT group was approximately equal to or greater than that of inpatient group. Besides, the total costs in DCT group (\$ 915.1) were lower than inpatient group (\$1227.9). Therefore, DCT was more cost effective than inpatient surgery and there was no need to calculate ICER. On the other hand, there was less blood transfusion in the inpatient group, thus, effectiveness of inpatient group (0.993) was approximately equal to or greater than that of DCT group (0.987) with no significant difference between two groups. However, the mean total costs in DCT group (\$915.1) were lower than inpatient group $(\$$ 1227.9). Thus, the calculated ICER was $\$ 52133$, indicating that per less blood transfusion in tonsillectomy by inpatient method, $\$ 52133$ should be spent. Thus, because the ICER is greater than the per capita GDP, DCT was more cost effective than inpatient method. Also, according to the Pain, patients in DCT group endured less pain. Therefore, the effectiveness of DCT group (0.730) was greater than inpatient group (0.710) and due to the lower total cost in the amount of pain of DCT group (\$915.1) compared with inpatient group $(\$ 1227.9)$, DCT was more cost effective and there was no need to calculate ICER.

\section{Financial burden and cost saving}

Annually, 2350 tonsillectomy is performed in DK* Hospital. If we consider only DCT in both hospitals, it would cost $\$ 2150575.2$, and if consider only inpatient tonsillectomy for all 2350 patients per year, it would cost $\$ 2885729.2$. The difference of financial burden for two methods was estimated as $\$ 735153.8$ for the 2,350 patients undergoing tonsillectomy in DK* Hospitals for a year. Cost-saving of DCT for patients underwent tonsillectomy in these hospitals may be increased about $\$ 735153.8$ for one year which could be saved by DCT.

\section{Sensitivity analysis}

To validate the results a one-way sensitivity analysis was performed. The value of variables changed by $20 \%$ and the tornado diagram was plotted. As shown in Figures 1, ICER in clinical outcomes including patients pain score, incidence of bleeding, incidence of blood transfusion and re-operation had the highest sensitivity to the inpatient effectiveness and DCT effectiveness and the lowest level of sensitivity to the cost of DCT.

\section{Discussion:}

To our knowledge, this research was the first comprehensive study on the cost-effectiveness of DCT and inpatient method in south of Iran.

Among all four clinical outcomes, DCT was considered as the most cost effective option. Mahdavan et al. studied on a total of 5400 tonsillectomies in children aged 7.4 months to 15 years with a mean age of 6.2 years and they observed no significant difference in bleeding after tonsillectomy between day case and hospitalized patients(17). Other studies, also support this $(15,16,17)$. Al-Sobaki et al (2014) showed that the pediatric patients who had day case surgery had no complications during the first 24 hours after surgery. Also, the cost of hospitalization for children was $\$ 1971$, while it was less than half $(\$ 870)$ for DCT method. These figures reveal that pediatric tonsillectomy is performed as an economically justified DCT procedure and it is recommended for selected patients if it meets their criteria(18). The result of the above mentioned study supports the result of the present study. Also, consistent with the results of this study, a study in Germany (2017) showed that outpatient surgery saves potentially about \euro 213500,000 annually. However, inconsistent with the results of the current study, tonsillectomy is not recommended as an outpatient surgery in Germany due to economic reasons and medical quality(13). Jain et al (2004) studied on 269 patients in the United Kingdom. The average hospital cost per patient was $£ 768$ for DCT and $£ 1430$ for admission which means that DCT was about $46 \%$ cheaper(19). This is in line with the results of the current study. In a retrospective study by Paquette et al. on 4040 patients, the average cost of hospital was $\$ 12978$ while it was $\$ 6391$ for a day case surgery center. In other words, the average patient cost for day case surgery center was approximately $\$ 50$ cheaper. Besides, the patients treated in day case surgery center were younger and had fewer concurrent diseases than those treated in hospital (21) which seems to be confirmed by the results of the present study. 
ICER compared with many parameters was not much sensitive and confirms the robustness of the study results. Moreover, ICER was more sensitive to the effectiveness of inpatient and DCT but less sensitive to other parameters. For instance, for the outcome of patient pain score, although the parameter of inpatient effectiveness had $20 \%$ increase on the parameter by the most effect on ICER and it increased ICER to almost 800 dollars PPP, however, the new amount of ICER is still lower than threshold and DCT is dominant and more cost-effective. This has happened also for the incidence of bleeding and re-operation; because by increasing ICER to 1000 dollars, again due to being lower than threshold, DCT is considered dominant. Regarding the outcome of incidence of blood transfusion, DCT is the superior option confidently; because it turned ICER from positive to negative

Economic considerations regarding the safety of day case surgery in tonsillectomy can have a major impact at national level. Supporting this method is completely reasonable and has benefits for both patients and insurance companies. This method reduces waiting time for selective surgery, implements a reservation system, and introduces the treatment centers for patients which require significant changes in the manner of surgery.

This study had some strengths like: considering the total costs, including direct medical and non-medical costs as well as indirect costs. One of the limitations of this study was lack of access to information of other hospitals in Iran.

Conclusion : It can be concluded that DCT is a cost-effective option and a superior strategy for our country, Iran, and can be suggested as a better alternative for a wide range of patients after tonsillectomy. According to the result of our study, supporting day case surgery for tonsillectomy cases can significantly reduce the financial burden.

\section{Declarations}

- Consent for publication Not applicable

- Availability of data and materials Datasets are available from the corresponding author on reasonable request.

- Competing interests

The authors declare that they have no competing interests.

Funding

Not applicable.

Figure legend:

Figure 1: Tornado diagram of Cost-effectiveness for patient's day case tonsillectomy (DCT) in comparison with inpatient tonsillectomy (to differ clinical outcomes)

\section{References}

1. Archer S, Rosenfeld R, Mitchell R, Baugh R. Clinical Practice Guideline: Tonsillectomy in Children. Otolaryngology-Head and Neck Surgery. 2010;143(2_suppl):P12-P.

2. Flint PW, Haughey BH, Niparko JK, Richardson MA, Lund VJ, Robbins KT, et al. Cummings OtolaryngologyHead and Neck Surgery E-Book: Head and Neck Surgery, 3-Volume Set: Elsevier Health Sciences; 2010.

3. Steward DL, Grisel J, Meinzen-Derr J. Steroids for improving recovery following tonsillectomy in children. Cochrane Database of Systematic Reviews. 2011(8).

4. Leong SC, Karkos PD, Papouliakos SM, Apostolidou MT. Unusual complications of tonsillectomy: a systematic review. American journal of otolaryngology. 2007;28(6):419-22. 
5. Rivas ML. Tonsillectomy as a major outpatient procedure. Prospective 8-year study: indications and complications. Comparison with inpatients. Acta otorrinolaringologica espanola. 2000;51(3):221-7.

6. Rakover Y, Almog R, Rosen G. The risk of postoperative haemorrhage in tonsillectomy as an outpatient procedure in children. International journal of pediatric otorhinolaryngology. 1997;41(1):29-36.

7. Bain J, Kelly H, Snadden D, Staines H. Day surgery in Scotland: patient satisfaction and outcomes. BMJ Quality \& Safety. 1999;8(2):86-91.

8. Jarrett P. Day care surgery. European Journal of Anaesthesiology. 2001;18(S23):32-5.

9. p l, P J, b. p. Day surgery.Development and practice 2006 [Available from: Http://www.iassmed.com/files/historical/day surgery.

10. Castoro C, Bertinato L, Baccaglini U, Drace CA, McKee M, Organization WH. Day surgery: making it happen. Copenhagen: WHO Regional Office for Europe; 2007.

11. Toftgaard C. World wide day surgery activity 2003: IAAS Survey of Ambulatory Surgery. Ambul Surg. 2007;13(5-24):11-61.

12. Bevan H, Robert G, Bate P, Maher L, Wells J. Using a Design Approach to Assist Large-Scale Organizational Change: "10 High Impact Changes" to Improve the National Health Service in England. The Journal of Applied Behavioral Science. 2007;43(1):135-52.

13. Schuldt T. Inpatient vs. outpatient costs analysis of the tonsillectomy. Laryngo-Rhino-Otologie. 2013;93(2):107-14.

14. Brazier J, Green C, McCabe C, Stevens K. Use of visual analog scales in economic evaluation. Expert review of pharmacoeconomics \& outcomes research. 2003;3(3):293-302.

15. Bank W. World Development Indicators [Available from: http://databank.worldbank.org/data/reports.aspx?source=2\&s

16. Masoom A, Akhtar S, Humayun HN, Ikram M. Daycare adeno-tonsillectomy: is it safe in developing countries. Journal of the Pakistan Medical Association. 2012;62(5):458.

17. Mahadevan M, van der Meer G, Gruber M, Reed P, Jackson C, Brown C, et al. The starship children's hospital tonsillectomy: A further 10 years of experience. The Laryngoscope. 2016;126(12):E416-E20.

18. Elsobky S, Ahmad N, Qureshi M, Izzath W, Sadiq H. Paediatric day case tonsillectomy: a safe, feasible and an economical way to treat patients-Yorkhill experience. Scottish medical journal. 2014;59(1):5-8.

19. Jain P, Hayden J, Sedman P, Royston C, O’Boyle C. A prospective study of ambulatory laparoscopic cholecystectomy: training economic, and patient benefits. Surgical Endoscopy And Other Interventional Techniques. 2005;19(8):1082-5.

\section{Hosted file}

Figure 1.docx available at https://authorea.com/users/310058/articles/440881-day-case-versusinpatient-tonsillectomy-in-southern-iran-a-cost-effectiveness-study

\section{Hosted file}

Tables.docx available at https://authorea.com/users/310058/articles/440881-day-case-versus-inpatienttonsillectomy-in-southern-iran-a-cost-effectiveness-study 